\title{
Heterozygous carriage of the alpha1-antitrypsin PiZ variant increases the risk to develop liver fibrosis
}

Pavel Strnad ${ }^{1,2 *}$, Stephan Buch ${ }^{3 *}$, Karim Hamesch ${ }^{1,2 *}$, Janett Fischer ${ }^{4}$, Jonas Rosendahl ${ }^{4,5}$, Renate Schmelz ${ }^{3}$, Stefan Brueckner ${ }^{3}$, Mario Brosch ${ }^{3}$, Carolin V. Heimes ${ }^{1}$, Marla Gutberlet ${ }^{1}$, David Scholten ${ }^{1}$, Hans Dieter Nischalke ${ }^{6}$, Sabina Janciauskiene ${ }^{7}$, Mattias Mandorfer ${ }^{8}$, Michael Trauner ${ }^{8}$, Michael J. Way, ${ }^{9,10}$, Andrew McQuillin ${ }^{10}$, Matthias Reichert ${ }^{11}$, Marcin Krawczyk ${ }^{11,12}$, Markus Casper ${ }^{11}$, Frank Lammert ${ }^{11}$, Felix Braun ${ }^{13}$, Witigo von Schoenfels ${ }^{13}$, Sebastian Hinz $^{13}$, Greta Burmeister ${ }^{13}$, Claus Hellerbrand ${ }^{14}$, Andreas Teufel ${ }^{15}$, Monika Ridinger ${ }^{16}$, Norbert Wodarz ${ }^{17}$, Michael Soyka ${ }^{18}$, Marcella Rietschel ${ }^{19}$, Falk Kiefer ${ }^{19}$, Thomas Weber ${ }^{20}$, Silke Marhenke ${ }^{21}$, Arndt Vogel21, Holger Hinrichsen ${ }^{22}$, Ali Canbay²3,24, Martin Schlattjan ${ }^{24}$, Katharina Sosnowsky ${ }^{25}$, Christoph Sarrazin ${ }^{25}$, Johann von Felden ${ }^{26}$, Pierre Deltenre ${ }^{27,28,}$ Bence Sipos ${ }^{29}$, Clemens Schafmayer ${ }^{13}$, Michael Nothnagel ${ }^{30}$, Elmar Aigner ${ }^{31}$, Christian Datz ${ }^{32}$, Felix Stickel ${ }^{33}$, Marsha Y. Morgan ${ }^{9}$, Jochen Hampe ${ }^{3+}$, Thomas Berg ${ }^{4+\#}$, Christian Trautwein ${ }^{1,2+}$

${ }^{1}$ Medical Clinic III, Gastroenterology, Metabolic diseases and Intensive Care, University Hospital RWTH Aachen, Aachen, Germany;

${ }^{2}$ Coordinating center for alpha1-antitrypsin deficiency-related liver disease of the European Reference Network (ERN) "Rare Liver" and the European Association for the Study of the Liver (EASL) registry group "Alpha1-Liver";

${ }^{3}$ Medical Department 1, University Hospital Dresden, TU Dresden, Dresden, Germany;

${ }^{4}$ Section of Hepatology, Department of Internal Medicine, University Hospital Leipzig, Leipzig, Germany;

${ }^{5}$ Department of Internal Medicine I, University Hospital Halle, Martin Luther University Halle, Germany;

${ }^{6}$ Department of Internal Medicine I, University of Bonn, Bonn, Germany;

${ }^{7}$ Clinic for Pneumology, German Center for Lung Research (DZL), Medical University Hannover, Hannover, Germany;

${ }^{8}$ Clinic for Gastroenterology und Hepatology, Medical University Vienna, Vienna, Austria;

9 Institute for Liver \& Digestive Health, Division of Medicine, Royal Free Campus, University College London, London, UK;

${ }^{10}$ Molecular Psychiatry Laboratory, Division of Psychiatry, University College London, London, UK;

${ }^{11}$ Department of Medicine II, Saarland University Medical Center, Homburg, Germany;

12 Laboratory of Metabolic Liver Diseases, Department of General, Transplantation and Liver Surgery, Medical University of Warsaw, Warsaw, Poland; 
${ }^{13}$ Department of General and Thoracic Surgery, University Hospital Schleswig-Holstein, Kiel, Germany;

${ }^{14}$ Institute of Biochemistry, Friedrich-Alexander University Erlangen-Nürnberg, Erlangen, Germany;

${ }^{15}$ Department of Internal Medicine I, University Hospital Regensburg, Regensburg, Germany;

${ }^{16}$ Department of Psychology, University of Konstanz, Konstanz, Germany;

17 Department of Psychiatry and Psychotherapy, University of Regensburg, Regensburg, Germany;

${ }^{18}$ Psychiatric Hospital, Ludwig Maximilians University, Munich, Germany;

${ }^{19}$ Central Institute of Mental Health, University of Heidelberg, Faculty of Medicine Mannheim, Mannheim, Germany;

20 Department for Clinical Research, University Hospital Bern, Bern, Switzerland;

${ }^{21}$ Department of Gastroenterology, Hepatology and Endocrinology, Hannover Medical School, Hannover, Germany;

22 Department of Gastroenterology, University Hospital Kiel, Kiel, Germany;

${ }^{23}$ Department of Gastroenterology, Hepatology and Infectious Diseases, Otto-von-Guericke University, Magdeburg, Germany;

${ }^{24}$ Department of Gastroenterology and Hepatology, University Duisburg-Essen, Essen, Germany;

${ }^{25}$ Department of Internal Medicine 1, J.W. Goethe University Hospital Frankfurt, Frankfurt, Germany;

${ }^{26}$ I. Department of Internal Medicine, University Medical Center Hamburg-Eppendorf, Hamburg, Germany;

${ }^{27}$ Division of Gastroenterology and Hepatology, Centre Hospitalier Universitaire Vaudois, University of Lausanne, Lausanne, Switzerland;

${ }^{28}$ Department of Gastroenterology, Hepatopancreatology and Digestive Oncology, CUB Hôpital Erasme, Université Libre de Bruxelles, Brussels, Belgium;

${ }^{29}$ Institute of Pathology, University of Tuebingen, Tuebingen, Germany;

${ }^{30}$ Cologne Center for Genomics, University of Cologne, Cologne, Germany;

${ }^{31}$ Department of Internal Medicine I, University Hospital Salzburg, Salzburg, Austria;

32 Department of Internal Medicine, Hospital Oberndorf, Teaching Hospital of the Paracelsus Private University of Salzburg, Oberndorf, Austria;

${ }^{33}$ Department of Gastroenterology and Hepatology, University Hospital of Zurich, Zurich, Switzerland;

*,+ The authors contributed equally.

\# To whom correspondence should be addressed: thomas.berg@medizin.uni-leipzig.de 


\section{Corresponding author:}

Thomas Berg, MD

Section of Hepatology, Department of Internal Medicine, University Hospital Leipzig, Leipzig, Germany

Address: Liebigstr. 20, 04103 Leipzig, Germany

Email: Thomas.berg@medizin.uni-leipzig.de

Phone: +49 $34197-12330$

\section{Abbreviations:}

AAT Alpha1-antitrypsin

AATD Alpha1-antitrypsin deficiency

PiMZ $\quad$ AAT genotype with heterozygosity for the PiZ variant

PiZZ AAT genotype with homozygosity for the PiZ variant

PiMS AAT genotype with heterozygosity for the PiS variant

PiSS AAT genotype with homozygosity for the PiS variant

PiSZ AAT genotype with compound heterozygosity for the PiS and the PiZ variant

PiMM $\quad$ AAT wild-type genotype

NAFLD Non-alcoholic fatty liver disease

ALD Alcohol-related liver disease

TE Transient elastography (FibroScan)

Word count: 295 (Abstract) +4698 (Manuscript)

Figures: 6 (2 possibly as supplement)

Tables: 5 (4 possibly as supplement) 


\section{Declarations:}

Declaration of competing interests: All authors declare: no support from any organisation other than the below mentioned ones for the submitted work; no financial relationships with any organisations that might have an interest in the submitted work in the previous three years; no other relationships or activities that could appear to have influenced the submitted work. Hence, all authors declare themselves to be independent from funders with respect to this manuscript.

Declaration of funding interests: This work was supported by the EASL registry grant on alpha1antitrypsin-related liver disease (to P.S. and C.T.), the Interdisciplinary Center for Clinical Research (IZKF) within the faculty of Medicine at the RWTH Aachen University (to P.S.), the Else Kröner Excellence Fellowship (to P.S.), the Deutsche Forschungsgemeinschaft (DFG) consortium SFB/TRR57 "Liver fibrosis" (to P.S. and C.T.), the German Liver Foundation (to K.H.), the German Ministry of Education and Research through the Virtual Liver Network (to J.H.), the Swiss National Funds (grant 310030_138747 to F.S.), the Deutsche Krebshilfe (to H.D.N), the Liver Systems Medicine (BMBF LiSyM 031L0051 to F.L. and C.T.), the Joseph-Skoda Award of the Austrian Society of Internal Medicine (to M.M.), the European Union $7^{\text {th }}$ framework programme (/FP7/2007-2013, project "FLIP" to M.T.). The British-Irish research effort was funded by a PhD studentship award jointly funded by University College London and an anonymous donor (to M.Y.M). The NAFLD cohort was supported by the German Ministry of Education and Research through the Virtual Liver and Liver Systems Medicine (LiSyM) Projects and through institutional funds from the Medical Faculty of the University of Kiel (to C.S.).

Further remarks: We attest that we did not use any copyright protected material in our manuscript.

Acknowledgements: We thank the national patient organisations (i.e., Alpha1 Deutschland, Alpha1 Oesterreich, Alpha1 Plus Belgium, Alpha1 Nederland) for their help with the execution of our study. We also thank all patients and their relatives for their participation in our registry. 


\section{Authorship statement:}

Study concept and design: P.S., K.H., F.S., M.Y.M., J.H., T.B., C.T.

Acquisition of data: P.S., S.B., K.H., J.R., J.F., R.S., S.B., M.B., C.V.H., M.G., D.S., H.D.N., M.M., M.T., M.J.W., A.M., M.R., M.K., F.L., F.B., W.V.S., C.H., A.T., M.R., N.W., M.S., M.R., F.K., T.W., S.M., A.V., H.H., A.C., M.S., K.S., C.S., J.V.F., S.H., G.B., P.D., B.S., C.S., E.A., C.D., F.S., M.Y.M, J.H., T.B.

Analysis and interpretation of data: P.S, S.B., K.H., S.J., M.M., B.S., M.N., E.A., M.Y.M., J.H., C.T.

Drafting of the manuscript: P.S., K.H., S.J., M.Y.M., J.H., T.B., C.T.

Figures and tables: P.S., S.B., K.H., M.Y.M., C.T.

Obtained funding: P.S., K.H., H.D.N., F.L., M.M., M.T., C.S., F.S., M.Y.M., J.H., C.T.

Study supervision: P.S., S.B., K.H., E.A., F.S., M.Y.M, J.H., T.B., C.T.

All authors had full access to all of the data and approved the final version of this manuscript. All authors can take responsibility for the integrity of the data and the accuracy of the data analysis. 


\section{What this paper adds}

\section{What is already known on this subject?}

- Alpha1-antitrypsin (AAT) deficiency is among the most common genetic diseases. Homozygous carriers of the 'PiZ' variant of AAT are highly susceptible to develop liver cirrhosis due to 'gain-offunction' toxicity of the mutant protein in hepatocytes.

- Up to $10 \%$ of Caucasians carry an AAT variant but the relevance of heterozygous carriage for developing cirrhosis remains unclear.

\section{What this study adds}

- Heterozygous PiZ carriers (PiMZ) have markedly higher odds for developing liver fibrosis, especially in the setting of alcohol misuse or metabolic liver injury.

- This finding warrants appropriate testing for the PiZ variant as a part of liver workup and a more thorough clinical monitoring of PiMZ carriers. 


\section{Abstract}

Objectives: Homozygous carriers of the alpha1-antitrypsin (AAT) 'PiZ' variant are at risk for developing liver cirrhosis, whereas the relevance of heterozygous carriage remains unclear. Hence, we evaluated (i) the prevalence of liver disease in heterozygous carriers of the PiZ variant ('PiMZ'); and (ii) the impact of AAT variants on subjects with non-alcoholic fatty liver disease (NAFLD) or alcohol misuse.

Design: Case-control study.

Setting: Multinational.

Participants: (i) 176 PiMZ carriers (German-Austrian-Belgian-Dutch) and 176 non-carriers without AAT mutations. (ii) 643 people with NAFLD (German-Austrian) and 2462 people misusing alcohol (GermanSwiss \& British-Irish).

Main outcome measures: Development of liver fibrosis.

Results: (i) PiMZ carriers had significantly higher liver enzyme and transient elastography (TE) values than non-carriers. The PiMZ genotype and obesity acted as independent risk factors for increased TE values suggesting significant liver fibrosis. (ii) The PiMZ genotype was present in $13.2 \%$ of cirrhotic NAFLD patients but only in $2.5 \%$ of patients without fibrosis $(p<.0001)$. Accordingly, carriage of the PiZ variant predisposed NAFLD subjects to cirrhosis (adjusted $\mathrm{OR}=6.7$ [1.5 to 31.0]). Likewise, the PiMZ genotype was present in $6.2 \%$ of alcohol misusers with cirrhosis but only in $2.2 \%$ of alcohol misusers without significant liver injury $(p<.0001)$. Correspondingly, alcohol misusers carrying the $\mathrm{PiZ}$ variant were prone to develop cirrhosis (adjusted $\mathrm{OR}=5.8$ [2.9 to 11.7]). These odds remained significant after adjustment for the established genetic variants. In contrast, the PiS variant showed no association with NAFLD-related cirrhosis and only a weak association with alcohol-related cirrhosis (adjusted OR=1.5 [1.0 to 2.2$])$.

Conclusions: The PiZ variant is the hitherto strongest single nucleotide polymorphism-based risk factor for liver fibrosis. This finding needs to be incorporated into genetic counseling of affected individuals. As 2$4 \%$ of Caucasians are PiMZ carriers these data advocate for AAT testing as a part of liver workup.

Trial registration: NCT02929940

Keywords: Alpha1-antitrypsin deficiency, SERPINA1, SNP, alcoholic liver disease, non-alcoholic fatty liver disease, genetic liver disease 


\section{Introduction}

Cirrhosis is the end-stage of all chronic liver diseases and is the result of a scarring process that is termed liver fibrosis. Together with its detrimental sequelae, cirrhosis constitutes a major and growing cause for morbidity and mortality worldwide(1). Therefore, a better understanding of the underlying genetic risk factors is highly relevant. Alcohol-related and non-alcohol-related liver diseases (ALD/NAFLD) are major causes of cirrhosis and liver-related mortality $(2,3)$. Both diseases share the same genetic risk factors. These comprise variants in the genes of patatin-like phospholipase domain-containing protein 3 (PNPLA3), transmembrane 6 superfamily member 2 (TM6SF2), and membrane bound O-acyltransferase domain containing $7(M B O A T 7)(4,5)$. These genetic variants have been identified by genome-wide association studies that are well suited for analysis of common genetic variants. However, variants that cause monogenic disorders are often rare and the genome-wide association approach fails to uncover such less frequent variants. A potential way to circumvent this problem is to directly address the biological role of genetic variants with a proven pathogenic potential. At this point, mutations in alpha1-antitrypsin (AAT; SERPINA1 gene) are attractive candidates. AAT is one of the most abundant serum proteins and is produced predominantly within hepatocytes(6). The presence of SERPINA1 variants typically lead to decreased serum AAT concentrations giving rise to the systemic disease alpha1-antitrypsin deficiency (AATD). Severe AATD is characterized by very low AAT serum levels $(10-20 \%$ of normal), and constitutes a 'frequent rare disease' with an incidence between 1:2000 and 1:4000(6-8). Accordingly, severe AATD is the third most common genetic disorder leading to death worldwide( 7,9$)$. The lung and the liver are the organs most commonly affected and their involvement accounts for the high morbidity and mortality $(7,9)$.

The nomenclature of AAT variants is based on the mobility of the resulting protein in the electric field(6, 9). The most relevant variant is termed 'PiZ' ( $r$ 28929474) which has a prevalence of up to $8 \%$ in Northern Europe $(6,8)$. The somewhat less severe variant, termed 'PiS' (rs17580), is more common in Southern Europe, with a prevalence of up to $20 \%(6)$. In total, approximately 120 million people worldwide carry at least one PiZ or PiS allele(6, 8). 'PiMM' describes the wild-type genotype and 'PiZZ' (i.e., the homozygous PiZ carriage) constitutes the classic severe AATD form $(6,7)$. While presence of the PiZZ genotype precipitates the development of liver disease $(6,10)$, the impact of heterozygous PiZ carriage (PiMZ genotype) is controversial(9). Even less well established is the impact of heterozygous or homozygous PiS carriage (PiMS or PiSS genotype, respectively) on liver disease. 
Led by the a priori hypothesis that the presence of heterozygous AAT variants may spur the liver disease development in susceptible individuals, we evaluated the impact of heterozygous PiZ or PiS carriage in different settings. First, we studied a large cohort of heterozygous PiZ carriers. Secondly, we analysed well-described cohorts of patients with NAFLD and chronic alcohol misuse for the presence of both the PiZ and the PiS variant. 


\section{Methods}

\section{Cohort of PiMZ carriers and non-carriers}

A total of 352 adult carriers and non-carriers of the PiZ variant of self-reported European descent were recruited in the period from 1 April 2015 through 31 August 2017. None of the carriers and noncarriers was known to have liver disease and the primary objective was to assess their extent of liver disease.

Carriers $(n=176)$ were defined as individuals with heterozygous carriage of the alpha1-antitrypsin (AAT) PiZ variant ('PiMZ' genotype). While some PiMZ carriers were known to have AATD, others have been recruited from families with a known case but had not themselves been genotyped previously. Only participants with a proven PiMZ genotype regardless of the AAT serum level were included. All carriers were recruited in close collaboration with the German, Belgian, Dutch, and Austrian patient advocacy groups as well as with respiratory physicians with a specialist interest in AATD. This awareness campaign was spear-headed by the University Hospital Aachen which is the coordinating center for AATD-related liver disease in two European initiatives (The European Association for the Study of the Liver (EASL) registry group 'Alpha1-Liver' and The European Reference Network (ERN) 'Rare-Liver') and was supported via an AATD liver-related homepage, telephone hotline, a presence on social media, as well as contributions to patient-centered periodicals. Individuals listed in the German national AATD registry were also offered a participation in the program.

Non-carriers ( $\mathrm{n}=176)$ had no evidence of any AAT mutation ('PiMM' genotype or wild-type). They had been recruited as spouses of patients with known AATD or as participants in a 'liver awareness campaign' organized by the University Hospital Aachen, Germany which provided a 'liver check-up' for the general population.

The inclusion criteria were a valid assessment via transient elastography (TE; FibroScan, Echosens, Paris, France) of the liver and an ability to provide written informed consent. Main exclusion criteria were age $<18$ years and pregnancy. Study inclusion was offered at various sites and all comers have been examined. All examinations (questionnaires, clinical examination, blood sampling, and TE) were performed within the same day. Every participant filled out detailed, standardized questionnaires including information on demographic parameters, relevant comorbidities, alcohol consumption, etc. In all participants, the presence of a previously diagnosed liver disease has been excluded by personal 
interview (e.g., no established diagnosis of chronic liver disease and no history of liver resection or liver transplantation) and clinical examination. Moreover, in both carriers and non-carriers, a comprehensive laboratory workup was performed using routine assays approved for use in clinical routine. Chronic infection with hepatitis B and C was excluded serologically in all subjects. Serum levels of ferritin and transferrin saturation were determined to assess for the presence of hereditary hemochromatosis and individuals with an otherwise unexplained elevation of ferritin and transferrin saturation were excluded (1 PiMZ carrier, 1 PiMM non-carrier). Patients with self-reported excess alcohol consumption (>40g/d women, $>60 \mathrm{~g} / \mathrm{d}$ men) were excluded (1 PiMZ carrier, 1 PiMM non-carrier). Finally, patients with elevated serum alanine transaminase (ALT) or aspartate transaminase (AST) activity $>5 x$ of the sex-specific upper limit of normal (ULN) or serum alkaline phosphatase (ALP) $>2 x$ of sex-specific ULN at time of recruitment were excluded (1 PiMZ carrier and no PiMM non-carrier) since marked elevation of these values preclude a reliable measurement of liver stiffness by TE. TE is the best established method for non-invasive assessment of liver fibrosis $(11,12)$ and was performed according to published recommendations (e.g., exclusion of confounders such as non-fasting or the presence of hepatic inflammation)(12). Previously suggested cut-offs for liver stiffness measurements were used(12). All measurements had been carried out by experienced physicians and only subjects with at least ten successful measurements and an interquartile range $\leq 30 \%$ of median liver stiffness were evaluated. All participants provided a written informed consent and were treated according to Ethical guidelines of the 1975 Declaration of Helsinki. The study was approved by the Internal Review Board of the participating centers and was registered on ClinicalTrials.gov (NCT02929940).

\section{Cohort of NAFLD subjects}

A total of 643 cases and controls of self-reported European descent were recruited from multiple sites in Germany and Austria. In all subjects, presence of non-alcoholic fatty liver disease (NAFLD) was diagnosed using liver biopsy. The presence of fibrosis was assessed according to Kleiner classification (stage F0: no fibrosis; stage F1: perisinusoidal fibrosis to portal/periportal fibrosis; stage F2: perisinusoidal and portal/periportal fibrosis; stage F3: bridging fibrosis; and stage F4: cirrhosis). Exclusion criteria were laboratory or clinical evidence of autoimmune, viral (serology for hepatitis B and C as well as HIV), or hereditary causes (serum ceruloplasmin and ferritin indicating the presence of Wilson disease and hereditary hemochromatosis, respectively) of liver disease, and clinically relevant alcohol consumption (self-reported consumption of $>30 \mathrm{~g} / \mathrm{d}$ for men and $>20 \mathrm{~g} / \mathrm{d}$ for women). Moreover, subjects with a current or previous use of steatosis-inducing drugs (e.g., amiodarone, methotrexate) were also excluded. 
Cases $(n=68)$ were defined as patients with biopsy-proven NAFLD-related cirrhosis (stage F4). Controls ( $\mathrm{n}=362$ ) had histological NAFLD without evidence of liver fibrosis (stage F0). In all NAFLD subjects, the relationship between fibrosis stage and the observed PiZ and PiS risk genotypes was analysed separately.

A total of 359 German subjects were recruited during routine NAFLD workup at Leipzig, Frankfurt, Homburg, Kiel, and Hamburg and 284 Austrian subjects were recruited from 2,500 participants in the 'Salzburg Colon Cancer Prevention Initiative' study and were examined in Oberndorf and Salzburg. Liver samples were obtained percutaneously in patients undergoing liver biopsy for suspected NAFLD/NASH or intraoperatively in patients in whom an intraoperative liver biopsy was indicated on clinical grounds such as during scheduled liver resection, exclusion of liver malignancy during major oncologic surgery, or assessment of liver histology during bariatric surgery. Portions of this NAFLD cohort have been described previously(13-19). All patients gave their written informed consent for liver biopsy and genetic testing. The study protocol was approved by the responsible local ethics committee of participating centers, and all subjects consented to inclusion in the study.

\section{Cohort of alcohol misusers}

A total of 2462 cases and controls were recruited from multiple centers. Of these, 1798 subjects were of self-reported German/Swiss descent (cases=934, controls=864) whereas 664 subjects were of self-reported British/Irish descent (cases=317, controls=347).

Cases were defined as patients with alcohol-related cirrhosis (defined histologically or based on clinical, laboratory, endoscopic and imaging criteria) on a background of past and/or present alcohol consumption of at least $60 \mathrm{~g} / \mathrm{d}$ for women and $80 \mathrm{~g} / \mathrm{d}$ for men for more than 10 years, after exclusion of other common causes of cirrhosis. Controls had similar alcohol exposure but no evidence of advanced liver disease (based on clinical, laboratory, endoscopic and imaging criteria, non-invasive assessment of liver fibrosis or liver histology).

German-Swiss cohort: A total of 1798 alcohol misusers of self-reported German or Swiss descent were examined. Cases with alcohol-related cirrhosis were recruited from the gastroenterology and hepatology departments of the university hospitals in Dresden, Leipzig, Bonn, Regensburg, Erlangen, Luebeck, Homburg, and Hamburg (all in Germany) or in Bern and Lausanne (Switzerland). Controls without evident liver disease were recruited at psychiatry centers specialized in addiction medicine in 
Regensburg, Munich, and Mannheim (all in Germany). Past and present alcohol consumption was quantified through a face-to-face interview. All patients underwent clinical examination, standard laboratory testing, and abdominal ultrasound. Chronic infection with hepatitis B and C was excluded serologically in all subjects. Serum levels of ferritin and transferrin saturation were determined to rule out hereditary hemochromatosis, and neither clinical nor serological signs of autoimmune liver disease were present. Cases with alcohol-related cirrhosis were diagnosed on the basis of a history of alcohol dependence and histological examination of liver tissue; or compatible historical, clinical, laboratory, radiological, and endoscopic features. Controls were diagnosed on the basis of a history of alcohol dependence and the absence of significant liver injury on histology or else the absence of historical, clinical, radiological, or endoscopic features suggestive of significant liver injury either at presentation or during prolonged follow-up. Details of recruitment protocols were reported previously (20). All patients gave written informed consent, and the study received approval from the ethics committees of all participating centers.

British-Irish cohort: 664 alcohol misusers of self-reported British or Irish descent were recruited from the Department of Hepatology at the Royal Free Hospital, London. All had a history of prolonged alcohol misuse and fulfilled DSM-IV criteria for a diagnosis of alcohol dependence. All were examined by two experienced, senior clinicians for signs of liver injury. Blood was taken at the time of presentation for standard liver function tests; serological testing was undertaken for antibodies to hepatitis A-E, cytomegalovirus, Epstein-Barr virus, herpes simplex and varicella; mitochondrial, nuclear, smooth muscle and liver kidney autoantibodies; iron, total iron binging capacity, ferritin; copper, caeruloplasmin; AAT and tissue transglutaminase. All patients underwent abdominal ultrasound and/or abdominal CT/MRI, as indicated; all underwent routine upper GI endoscopy; histological examination was undertaken, whenever possible, of liver biopsy material obtained by percutaneous, ultrasound guided or transjugular routes; or else of explant or post-mortem liver tissue. Patients were excluded if they had any other potential cause of liver injury or if they had a BMI $>30 \mathrm{~kg} / \mathrm{m}^{2}$ or were diabetic. Patients with AAT serum levels below laboratory reference range were genotyped (UK reference laboratory in Sheffield) and they were excluded if they had the PiZZ genotype. Cases with alcohol-related cirrhosis were diagnosed on the basis of a history of alcohol dependence and histological examination of liver tissue; or compatible historical, clinical, laboratory, radiological, and endoscopic features. Controls were diagnosed on the basis of a history of alcohol dependence and the absence of liver injury on histology or else the absence of historical, clinical, radiological, or endoscopic features suggestive of significant liver injury either at presentation or during 
prolonged follow-up. Further details are stated elsewhere10. The study protocol was approved by the institutional review board, and all included subjects provided written informed consent.

\section{Genotyping}

All three cohorts were genotyped for the presence of the PiZ variant (rs28929474, also known as p.E342K or Glu342Lys) and the PiS variant (rs17580, also known as p.E264V or Glu288Val) of AAT. For the PiMZ carrier cohort, genomic DNA in participants without prior genotyping was genotyped by the German reference laboratory in Marburg as described before(21). For the NAFLD and alcohol misuser cohorts, genomic DNA from all cases and controls was genotyped using TaqMan chemistry (Applied Biosystems) as described before(20). Moreover, genotyping was performed for the PNPLA3 variant rs738409 (hcv7241), the TM6SF2 variant rs58542926 (hcv89463510), and the MBOAT7 variant rs641738 (hcv8716820). All process data was logged and administered using a database-driven LIMS(22).

\section{Statistical analyses}

Continuous variables were displayed as mean \pm standard deviation (SD) and were compared by an unpaired, two-tailed t-test as well as by a multivariate linear model to account for relevant confounders. Categorical variables were reported as absolute $(n)$ and relative (\%) frequencies and contingency tables were analysed with Chi-square test. Mantel-Haenzsel linear-by-linear test for trend was used to assess the relationship between advanced fibrosis stage and observed PiZ and PiS risk genotypes in NAFLD subjects. Associations between clinical outcome parameters (e.g., liver stiffness, presence of cirrhosis) and potential risk factors (e.g., genotype) were assessed by binary logistic regression analysis in order to calculate odds ratios (OR). ORs were given with their corresponding 95\% confidence intervals [in brackets]. Multivariable logistic regression was used to test for independent associations. Nominal twosided $\mathrm{p}$ values were reported for all tests and were corrected for multiple testing by Bonferroni correction, wherever appropriate. Fixed-effect model meta-analysis was performed using the inverse varianceweighted method to summarise PiZ and PiS effect sizes in different cohorts. Herefore, the program META (1.6.0) was used.

Differences were considered to be statistically significant when $p<0.05$. Statistical analyses were performed using SPSS version 23 (IBM, Armonk, NY, USA) and the graphs were created with Microsoft Office (Microsoft, Redmond, WA, USA) and Prism 5 (GraphPad, La Jolla, CA, USA). 
Patients were actively involved in the recruitment of the the PiMZ carrier/non-carrier cohort (details see above). In particular, we closely collaborated with various patient interest groups and offered examinations at various sites. As an appreciation of this joint effort, Pavel Strnad was elected as a member of the scientific advisory board of the German AATD patient advocacy group. Every study participant received a comprehensive evaluation as well as recommendations for further liver care. Additionally, study results were disseminated at AATD patient meetings in several European countries. 


\section{Results}

PiMZ carriers displayed an increased liver disease burden

To assess the liver disease burden in individuals with the heterozygous 'PiMZ' genotype, we recruited a cohort of PiMZ carriers and non-carriers without known liver disease. Both groups displayed similar age, sex distribution, BMI values, and rates of diabetes mellitus (Table 1). Relevant alcohol consumption ( $>12 \mathrm{~g} / \mathrm{d}$ for women/>24g/d for men) was infrequent in both groups (individuals with consumption of $>40 \mathrm{~g} / \mathrm{d}$ for women $/>60 \mathrm{~g} / \mathrm{d}$ for men were excluded upfront), but tended to be more common in non-carriers (Table 1). As expected, PiMZ carriers had markedly lower serum AAT concentrations compared to non-carriers (Fig. 1A). The previously suggested cut-off AAT level of $110 \mathrm{mg} / \mathrm{dL}(23)$ discriminated well between both groups. Serum liver enzyme activities were increased above the sexadjusted upper limit of normal (ULN) in only a minority of PiMZ carriers: alanine aminotransferase (ALT): 15.4\%, aspartate aminotransferase (AST): $5.1 \%$, and gamma-glutamyltransferase (GGT): $16.6 \%$. The mean serum ALT, AST, and GGT activities were higher in PiMZ carriers than non-carriers (76\% vs. $65 \%$ of ULN, $66 \%$ vs. $61 \%$ of ULN, and $78 \%$ vs. $55 \%$ of ULN, respectively; $p<0.05$ for all; Fig. 1 B-D). In contrast, the cholestatic markers serum alkaline phosphatase (ALP) and serum bilirubin were not different between the groups (Fig. 1E-F). A multivariate adjustment of serum AAT, ALT, AST, GGT, ALP, and bilirubin for the covariates sex, age, BMI, and the presence of diabetes mellitus confirmed that the associations of serum AAT, ALT, AST and GGT concentrations with the genotype were independent $(p<0.05$ for all).

Transient elastography (TE) revealed that $12.0 \%$ of PiMZ carriers vs. $4.5 \%$ of non-carriers displayed liver stiffness values $\geq 7.1 \mathrm{kPa}$, suggesting the presence of significant liver fibrosis (fibrosis stage of at least 2) (unadjusted $\mathrm{OR}=2.95[95 \% \mathrm{Cl} 1.25$ to 6.90$], p=0.013$ ). This association was more pronounced after adjusting for sex, age, $\mathrm{BMI}$, and diabetes ( $\mathrm{OR}=3.79$ [1.47 to 9.77$], p=0.006)$ and after further adjustment for mean alcohol consumption ( $\mathrm{OR}=3.79$ [1.47 to 9.79], $p=0.006)$. In line, the average TE values were significantly higher in PiMZ carriers compared to non-carriers $(5.0 \pm 1.7$ vs. $4.4 \pm 1.4 \mathrm{kPa}$, univariate analysis: $p=.0004$, multivariate analysis accounting for age, sex, BMI, and diabetes: $p=.0003$; Fig. 2A).

The mean $( \pm 1 \mathrm{SD})$ TE values in non-obese patients $\left(\mathrm{BMl}<30 \mathrm{~kg} / \mathrm{m}^{2}\right)$ were normal irrespective of carrier status (PiMZ $4.7 \pm 1.4$ vs. PiMM 4.3 $\pm 1.3 \mathrm{kPa}, p=0.047$; Fig. $2 \mathrm{~B}$ ). The mean TE value in obese carriers was significantly higher than in non-carriers ( $\mathrm{PiMZ} 6.4 \pm 1.9$ vs $\mathrm{PiMM} 5.4 \pm 1.5 \mathrm{kPa}, p=0.043$; Fig. 
2B). Thus, TE values $\geq 7.1 \mathrm{kPa}$ were uncommon in non-obese individuals regardless of their genotype (PiMM 3/143 [2.1\%], PiMZ 9/137 [6.6\%]), but were more common in obese subjects (PiMM 5/33 [15.2\%], PiMZ 12/39 [30.8\%]). Accordingly, obesity represented a risk factor for TE values $\geq 7.1 \mathrm{kPa}$ in both genotypes (unadjusted odds: PiMM OR=8.87 [1.99 to 39.49], $p=0.004$; PiMZ OR=7.64 [2.80 to 20.85], $p<0.0001)$. This association remained significant after adjustment for sex, age, diabetes, and alcohol consumption (PiMM: OR=7.07 [1.42 to 35.14], p=0.017; PiMZ: OR=9.75 [3.23 to 29.42], $p<0.0001$ ). Consecutive inclusion and testing of PiMZ genotype carriership, obesity $\left(\mathrm{BMI} \geq 30 \mathrm{~kg} / \mathrm{m}^{2}\right)$ and their interaction term in a logistic regression model on the status of TE values $\geq 7.1 \mathrm{kPa}$, confirmed significant contributions by both PiMZ $(p=0.02)$ and obesity $(p<0.001)$. Their interaction (PiMZ $x$ obesity) turned out to be non-significant $(p=0.76)$ when subsequently added to this model. Consideration of BMI instead of dichotomized obesity status yielded very similar results, with an even stronger effect for PiMZ (OR=3.46 [1.37 to 8.70], $p=0.008)$. Hence, PiMZ and obesity act independently of each other as risk factors for the development of significant liver fibrosis. Collectively, these data indicate that the PiMZ genotype increases the risk of liver disease progression in the presence of a second hit.

\section{PiZ variant increased the risk to develop cirrhosis in NAFLD}

The increased liver stiffness in PiMZ carriers prompted us to investigate the role of AAT variants as disease modifier in patients with a second hit. First, we focused on patients with non-alcoholic fatty liver disease (NAFLD). Here, we genotyped a German-Austrian cohort of 643 patients with biopsy-proven NAFLD for the presence of the PiZ and the PiS variants (Supplementary table 1). No patient had the homozygous PiSS or PiZZ genotype or the compound heterozygous PiSZ genotype. A comparison of carriers of the PiMZ and PiMS genotype with non-carriers revealed that PiMZ carriers but not PiMS carriers displayed a significant shift towards higher fibrosis stages $(p=0.001$ and $p=0.320$, respectively; Supplementary table 1).

Noteworthy, the PiMZ genotype was overrepresented in NAFLD patients with cirrhosis vs. no fibrosis $(13.2 \%$ vs. $2.5 \%, p<0.0001$; Supplementary table 2$)$, while the PiMS genotype was detected at comparable frequencies $(4.4 \%$ vs. $3.9 \%, p=0.740$; Supplementary table 2$)$.

The PiZ variant constituted a strong risk factor for developing NAFLD-related cirrhosis (F4) vs. no fibrosis (F0) in the univariate analysis (unadjusted $\mathrm{OR}=5.76$ [1.82 to 18.24], $p=0.003$; Fig. 3A). This association remained significant after multivariate adjustment for sex, age, $\mathrm{BMI}$, and diabetes $(\mathrm{OR}=6.73$ [1.46 to 30.99], $p=0.014$; Fig. 3B). 
In contrast to the PiZ variant, no link with cirrhosis development was found for the PiS variant, neither in the univariate analysis ( $O R=1.71$ [0.37 to 8.05], $p=0.495$; Supplementary fig. $1 \mathrm{~A})$, nor after adjusting for sex, age, BMI, and diabetes (adjusted $\mathrm{OR}=1.77$ [0.32 to 9.83], $p=0.516$; Supplementary fig. 1B).

Taken together, the presence of the PiZ variant, but not the PiS variant, constitutes a strong risk factor to develop NAFLD-related cirrhosis.

\section{PiZ variant increased the risk to develop cirrhosis in alcohol misusers}

The link between the PiZ variant and the development of liver disease in NAFLD patients prompted us to investigate the impact of the same two AAT variants, PiZ and PiS, in two wellcharacterized cohorts of alcohol misusers, which in total comprised 2462 individuals (German-Swiss cohort, Supplementary table 3 , and British-Irish cohort, Supplementary table 4). Similar to the NAFLD cohort, the PiMZ genotype was overrepresented amongst patients with alcohol-related cirrhosis compared to controls (German-Swiss: $6.2 \%$ vs. $2.0 \%, p<0.0001$, Supplementary table 3; British-Irish: $6.0 \%$ vs. $2.6 \%$, $p=0.029$, Supplementary table 4 ; both cohorts: $6.2 \%$ vs. $2.2 \%, p<0.0001)$. The PiMS genotype was also overrepresented amongst patients with alcohol-related cirrhosis in the German-Swiss cohort compared to controls $(7.9 \%$ vs. $5.5 \%, p=0.046$; Supplementary table 3$)$ but not in the British-Irish cohort $(10.5 \%$ vs. $8.2 \% ; p=0.295$; Supplementary table 4). Notably, we also identified four subjects with previously unknown homozygous PiZ carriage (PiZZ genotype) in the German-Swiss cohort as measurement of the AAT serum level was not part of the initial assessment. Moreover, eight patients were newly discovered as compound heterozygotes (PiSZ genotype). Eleven out of the twelve PiZZ and PiSZ patients had already developed cirrhosis (Supplementary tables 3 and 4). Five patients were homozygous for the PiS variant (PiSS genotype); three of them had cirrhosis (Supplementary tables 3 and 4).

In line with these data, the presence of the PiZ variant was strongly associated with the risk of developing cirrhosis in alcohol misusers in the univariate analysis (German-Swiss: OR=5.35 [2.53 to 11.34], $p<0.0001$; British-Irish: $\mathrm{OR}=4.88$ [1.42 to 16.76 ], $p=0.012$; meta-analysis: $\mathrm{OR}=5.22$ [2.75 to 9.91 ], $p<0.0001$; Fig. 4A). This association was more pronounced when the data were adjusted for sex, age, BMI, and diabetes (German-Swiss: OR=6.14 [2.63 to 14.31], $p=<0.0001$; British-Irish: $\mathrm{OR}=5.11$ [1.47 to 17.81], $p=0.010$; meta-analysis: $\mathrm{OR}=5.79$ [2.87 to 11.68 ], $p=<0.0001$; Fig. $4 \mathrm{~B}$ ). Moreover, this association remained significant after further adjusting for risk variants in PNPLA3, TM6SF2, and MBOAT7, which are 
well-established ALD modifiers (German-Swiss: OR=6.05 [2.54 to 14.43], $p<0.0001$; British-Irish: OR=5.24 [1.45 to 18.85 ], $p=0.011$; meta-analysis: $\mathrm{OR}=5.78$ [2.82 to 11.87 ], $p<0.0001)$.

In contrast to the PiZ variant, no or only a weak association was observed between the PiS variant and the risk of developing cirrhosis in the univariate analysis (German-Swiss: OR=1.79 [1.15 to 2.79], $p=0.010$; British-Irish: $\mathrm{OR}=1.10$ [0.60 to 2.01], $p=0.750$; meta-analysis: $\mathrm{OR}=1.51$ [1.06 to 2.15], $p=0.024$; Supplementary fig. 2A). After multivariate adjustment for sex, age, BMI, and diabetes, this association remained significant in the German-Swiss cohort but lost its significance in the meta-analysis (German-Swiss: $\mathrm{OR}=1.70$ [1.01 to 2.87], $p=0.047$; British-Irish: $\mathrm{OR}=1.20$ [0.65 to 2.22], $p=0.557$; metaanalysis: $\mathrm{OR}=1.47$ [0.99 to 2.19], $p=0.058$; Supplementary fig. $2 \mathrm{~B}$ ). Moreover, after further adjustment for the variants in the PNPLA3, TM6SF2, and MBOAT7 genes this association was lost (German-Swiss: $\mathrm{OR}=1.56$ [0.89 to 2.74], $p=0.123$; British-Irish: $\mathrm{OR}=1.21$ [0.62 to 2.36], $p=0.570$; meta-analysis: $\mathrm{OR}=1.40$ [0.91 to 2.16], $p=0.122)$.

Altogether, our data indicate that carriage of the PiZ variant represents a strong factor predisposing to develop liver fibrosis, whereas the PiS variant does not confer a major risk. 


\section{Discussion}

In the present study, for the first time, we prospectively evaluated liver injury and the stage of liver fibrosis in PiMZ carriers. The fact that PiMZ subjects display higher liver enzyme levels and higher liver stiffness than those without AAT mutations does suggest that PiMZ carriers either have subclinical liver disease and/or are more susceptible to develop liver disease after a second hit. In addition to our genetic association data, the increased TE values found in obese PiMZs support the second-hit theory. With regard to that, the detrimental role of obesity for liver injury in general is well established(24). Obesity was also shown to predispose patients with severe AATD (homozygous PiZZ genotype) to advanced liver disease(25).

To further substantiate the data from the PiMZ cohort, we took advantage of a well-defined cohort of patients with biopsy-proven NAFLD ( $n=643)$ and two large, well-characterized cohorts of alcohol misusers $(n=2462)$. We unambiguously found that the PiZ variant is a major risk factor for ALD- and NAFLD-related cirrhosis. These findings are in line with previously published, smaller studies(26-29). Moreover, this study is the first report that has systematically investigated the role of the PiS variant providing evidence that this variant may also pose a weak risk to develop alcoholic cirrhosis, but further studies are needed to confirm this association. These observations mesh well with the known pathogenic significance of both PiS and PiZ variants. Because PiS forms fewer polymers than PiZ, it is less retained within hepatocytes leading to a less prominent endoplasmic reticulum (ER) protein overload than $\mathrm{PiZ}(30)$.

Our findings demonstrate that the PiZ variant confers an approximately six times higher odd to develop both NAFLD- and ALD-associated cirrhosis. With a frequency of 2-4\% in Europe the PiZ variant is relatively rare $(6,8,9)$, but shows remarkable odds posing this variant as the hitherto strongest known genetic risk factor for NAFLD- and ALD-related cirrhosis. In fact, the odds ratio for developing cirrhosis of the PiZ variant exceed the odds ratios of other, well-established genetic risk factors, such as the PNPLA3 variant rs738409, the MBOAT7 variant rs641738, and the TM6SF2 variants rs10401969/rs58542926(20, 31-33).

While future research needs to define the exact molecular mechanism how AAT variants predispose for NAFLD- and ALD-related liver fibrosis and what clinical modifiers are of particular importance, several factors are likely relevant. The cellular retention of mutated AAT (such as the PiZ variant) leads to a gain-of-function toxicity that is proportional to the amount of retained protein(9). Notably, AAT constitutes an established acute phase protein that is induced in patients suffering from liver 
injury(34). Among other factors, ethanol was shown to stimulate AAT production in a human hepatoma cell line(35), and thus, might aggravate gain-of-function toxicity in patients with AATD. Additionally, AAT mutations lower the threshold for an ER stress response(36); which might be particularly relevant in NAFLD and ALD as both also challenge the $\operatorname{ER}(9,37)$. Moreover, autophagy is crucial for the degradation of mutated AAT(36) and both NAFLD and ALD have been shown to impair autophagic activity in hepatocytes(38, 39). Finally, an increased activity of neutrophil elastase, that constitutes the most established AAT substrate, has been implicated in the development of obesity and insulin resistance(40). This pathway might be particularly important in subjects with AAT variants that display decreased serum AAT levels(9).

The fact that the PiZ variant constitutes a clear and strong disease modifier should prompt its assessment in the workup of liver disease. In that respect, measuring serum AAT levels represents a relatively easy and cost-efficient method to rule out severe AATD (i.e., homozygous PiZZ genotype)(23) However, detecting heterozygous individuals (e.g., PiMZ genotype) is more challenging as their AAT serum level are often within the reference range. Further research is needed to define an optimal serum AAT cut-off level, especially in the context of chronic liver disease. In summary, our study defines the PiZ variant as an obvious single nucleotide polymorphism-based risk factor to develop NAFLD- and ALDassociated cirrhosis. At the same time, our data advocate for a careful assessment of AAT serum levels in patients with chronic liver disease. They also encourage a more thorough clinical monitoring and management of individuals with heterozygous $\mathrm{PiZ}$ carriage. 


\section{Data sharing}

Patient level data, technical appendix, and statistical code are available from the corresponding authors and can be shared upon request. An informed consent for data sharing was not obtained from study participants,but the presented data are anonymized and the risk of identification is low. 


\section{References}

1. Collaborators GBDMM. Global, regional, and national levels of maternal mortality, 1990-2015: a systematic analysis for the Global Burden of Disease Study 2015. Lancet. 2016;388(10053):1775-812.

2. Rehm J, Mathers C, Popova S, Thavorncharoensap M, Teerawattananon Y, Patra J. Global burden of disease and injury and economic cost attributable to alcohol use and alcohol-use disorders. Lancet. 2009;373(9682):2223-33.

3. Chalasani N, Younossi Z, Lavine JE, Diehl AM, Brunt EM, Cusi K, et al. The diagnosis and management of non-alcoholic fatty liver disease: practice guideline by the American Gastroenterological Association, American Association for the Study of Liver Diseases, and American College of Gastroenterology. Gastroenterology. 2012;142(7):1592-609.

4. Stickel F, Moreno C, Hampe J, Morgan MY. The genetics of alcohol dependence and alcohol-related liver disease. Journal of hepatology. 2017;66(1):195-211.

5. Anstee QM, Seth D, Day CP. Genetic Factors That Affect Risk of Alcoholic and Nonalcoholic Fatty Liver Disease. Gastroenterology. 2016;150(8):1728-44 e7.

6. Greene CM, Marciniak SJ, Teckman J, Ferrarotti I, Brantly ML, Lomas DA, et al. alpha1-Antitrypsin deficiency. Nature reviews Disease primers. 2016;2:16051.

7. Silverman EK, Sandhaus RA. Alpha1-Antitrypsin Deficiency. N Engl J Med. 2009;360:2749-57.

8. Blanco I, Bueno P, Diego I, Perez-Holanda S, Casas-Maldonado F, Esquinas C, et al. Alpha-1 antitrypsin $\mathrm{Pi}^{\star} \mathrm{Z}$ gene frequency and $\mathrm{Pi}^{\star} \mathrm{ZZ}$ genotype numbers worldwide: an update. Int $\mathrm{J}$ Chron Obstruct Pulmon Dis. 2017;12:561-9.

9. Strnad P, Nuraldeen R, Guldiken N, Hartmann D, Mahajan V, Denk H, et al. Broad spectrum of hepatocyte inclusions in humans, animals, and experimental models. Comprehensive Physiology. 2013;3(4):1393-436.

10. Eriksson S, Carlson J, Velez R. Risk of cirrhosis and primary liver cancer in alpha 1-antitrypsin deficiency. N Engl J Med. 1986;314(12):736-9.

11.Tapper EB, Lok AS. Use of Liver Imaging and Biopsy in Clinical Practice. N Engl J Med. 2017;377(8):756-68.

12.Friedrich-Rust M, Poynard T, Castera L. Critical comparison of elastography methods to assess chronic liver disease. Nature reviews Gastroenterology \& hepatology. 2016;13(7):402-11.

13.Feldman A, Eder SK, Felder TK, Kedenko L, Paulweber B, Stadlmayr A, et al. Clinical and Metabolic Characterization of Lean Caucasian Subjects With Non-alcoholic Fatty Liver. Am J Gastroenterol. 2017;112(1):102-10.

14.Stattermayer AF, Traussnigg S, Aigner E, Kienbacher C, Huber-Schonauer U, Steindl-Munda P, et al. Low hepatic copper content and PNPLA3 polymorphism in non-alcoholic fatty liver disease in patients without metabolic syndrome. J Trace Elem Med Biol. 2017;39:100-7.

15.Teufel A, Itzel T, Erhart W, Brosch M, Wang XY, Kim YO, et al. Comparison of Gene Expression Patterns Between Mouse Models of Nonalcoholic Fatty Liver Disease and Liver Tissues From Patients. Gastroenterology. 2016;151(3):513-25.e0.

16. Horvath S, Erhart W, Brosch M, Ammerpohl O, von Schonfels W, Ahrens M, et al. Obesity accelerates epigenetic aging of human liver. Proceedings of the National Academy of Sciences of the United States of America. 2014;111(43):15538-43. 
17.Eslam M, Mangia A, Berg T, Chan HL, Irving WL, Dore GJ, et al. Diverse impacts of the rs58542926 E167K variant in TM6SF2 on viral and metabolic liver disease phenotypes. Hepatology. 2016;64(1):3446.

18.Aigner E, Strasser M, Haufe H, Sonnweber T, Hohla F, Stadlmayr A, et al. A role for low hepatic copper concentrations in nonalcoholic Fatty liver disease. Am J Gastroenterol. 2010;105(9):1978-85.

19.Stadlmayr A, Aigner E, Steger B, Scharinger L, Lederer D, Mayr A, et al. Nonalcoholic fatty liver disease: an independent risk factor for colorectal neoplasia. J Intern Med. 2011;270(1):41-9.

20.Buch S, Stickel F, Trepo E, Way M, Herrmann A, Nischalke HD, et al. A genome-wide association study confirms PNPLA3 and identifies TM6SF2 and MBOAT7 as risk loci for alcohol-related cirrhosis. Nat Genet. 2015;47(12):1443-8.

21.Greulich T, Nell C, Herr C, Vogelmeier C, Kotke V, Wiedmann S, et al. Results from a large targeted screening program for alpha-1-antitrypsin deficiency: 2003 - 2015. Orphanet J Rare Dis. 2016;11(1):75.

22. Franke A, Wollstein A, Teuber M, Wittig M, Lu T, Hoffmann K, et al. GENOMIZER: an integrated analysis system for genome-wide association data. Hum Mutat. 2006;27(6):583-8.

23.Ferrarotti I, Poplawska-Wisniewska B, Trevisan MT, Koepke J, Dresel M, Koczulla R, et al. How Can We Improve the Detection of Alpha1-Antitrypsin Deficiency? PloS one. 2015;10(8):e0135316.

24.Williams R, Aspinall R, Bellis M, Camps-Walsh G, Cramp M, Dhawan A, et al. Addressing liver disease in the UK: a blueprint for attaining excellence in health care and reducing premature mortality from lifestyle issues of excess consumption of alcohol, obesity, and viral hepatitis. Lancet. 2014;384(9958):1953-97.

25.Bowlus CL, Willner I, Zern MA, Reuben A, Chen P, Holladay B, et al. Factors associated with advanced liver disease in adults with alpha1-antitrypsin deficiency. Clin Gastroenterol Hepatol. 2005;3(4):390-6.

26. Eigenbrodt ML, McCashland TM, Dy RM, Clark J, Galati J. Heterozygous alpha 1-antitrypsin phenotypes in patients with end stage liver disease. Am J Gastroenterol. 1997;92(4):602-7.

27.Regev A, Guaqueta C, Molina EG, Conrad A, Mishra V, Brantly ML, et al. Does the heterozygous state of alpha-1 antitrypsin deficiency have a role in chronic liver diseases? Interim results of a large casecontrol study. J Pediatr Gastroenterol Nutr. 2006;43 Suppl 1:S30-5.

28. Cacciottolo TM, Gelson WT, Maguire G, Davies SE, Griffiths WJ. Pi`Z heterozygous alpha-1 antitrypsin states accelerate parenchymal but not biliary cirrhosis. Eur J Gastroenterol Hepatol. 2014;26(4):412-7.

29.Goltz D, Hittetiya K, Vossing LM, Kirfel J, Spengler U, Fischer HP. Alpha(1)-antitrypsin PiMZ heterozygosity has an independent aggravating effect on liver fibrosis in alcoholic liver disease. Virchows Arch. 2014;465(5):539-46.

30. Mahadeva R, Chang WS, Dafforn TR, Oakley DJ, Foreman RC, Calvin J, et al. Heteropolymerization of $\mathrm{S}, \mathrm{I}$, and $\mathrm{Z}$ alpha1-antitrypsin and liver cirrhosis. The Journal of clinical investigation. 1999;103(7):999-1006.

31.Valenti L, Al-Serri A, Daly AK, Galmozzi E, Rametta R, Dongiovanni P, et al. Homozygosity for the patatin-like phospholipase-3/adiponutrin I148M polymorphism influences liver fibrosis in patients with nonalcoholic fatty liver disease. Hepatology. 2010;51(4):1209-17. 
32.Liu YL, Reeves HL, Burt AD, Tiniakos D, McPherson S, Leathart JB, et al. TM6SF2 rs58542926 influences hepatic fibrosis progression in patients with non-alcoholic fatty liver disease. Nature communications. 2014;5:4309.

33. Mancina RM, Dongiovanni P, Petta S, Pingitore P, Meroni M, Rametta R, et al. The MBOAT7-TMC4 Variant rs641738 Increases Risk of Nonalcoholic Fatty Liver Disease in Individuals of European Descent. Gastroenterology. 2016;150(5):1219-30.e6.

34.Carrell RW, Lomas DA. Alpha1-antitrypsin deficiency--a model for conformational diseases. N Engl J Med. 2002;346(1):45-53.

35.Weinstein JA, Vanderburg G. Ethanol induces a1-protease inhibitor mRNA in Hep 3B cells. J Surg Res. 1991;51(6):463-6.

36. Hidvegi T, Ewing M, Hale P, Dippold C, Beckett C, Kemp C, et al. An autophagy-enhancing drug promotes degradation of mutant alpha1-antitrypsin $Z$ and reduces hepatic fibrosis. Science. 2010;329(5988):229-32.

37. Malhi H, Kaufman RJ. Endoplasmic reticulum stress in liver disease. Journal of hepatology. 2011;54(4):795-809.

38. Baiceanu A, Mesdom P, Lagouge M, Foufelle F. Endoplasmic reticulum proteostasis in hepatic steatosis. Nat Rev Endocrinol. 2016;12(12):710-22.

39. Ueno T, Komatsu M. Autophagy in the liver: functions in health and disease. Nature reviews Gastroenterology \& hepatology. 2017;14(3):170-84.

40.Mansuy-Aubert V, Zhou QL, Xie X, Gong Z, Huang JY, Khan AR, et al. Imbalance between neutrophil elastase and its inhibitor alpha1-antitrypsin in obesity alters insulin sensitivity, inflammation, and energy expenditure. Cell metabolism. 2013;17(4):534-48. 


\section{Tables}

Table 1: Features of individuals with heterozygous carriage of the alpha1-antitrypsin PiZ variant (PiMZ) and non-carriers (PiMM).

\begin{tabular}{|c|c|c|c|}
\hline & $\begin{array}{l}\text { Non-carriers } \\
\text { (PiMM) } \\
n=176\end{array}$ & $\begin{array}{l}\text { Carriers } \\
\text { (PiMZ) } \\
n=176\end{array}$ & $\begin{array}{l}\text { Significance } \\
\text { ( } p \text { value) }\end{array}$ \\
\hline \multicolumn{4}{|l|}{ Demographics } \\
\hline Age (years) & $47.0 .0 \pm 13.4$ & $47.0 \pm 15.5$ & 0.956 \\
\hline Women & $104(59.1)$ & $104(59.1)$ & 1.000 \\
\hline \multicolumn{4}{|l|}{ Risk factors } \\
\hline $\mathrm{BMI}\left(\mathrm{kg} / \mathrm{m}^{2}\right)$ & $25.7 \pm 4.7$ & $25.7 \pm 4.5$ & 0.994 \\
\hline Diabetes mellitus (yes/no/NA, \% yes) & $6 / 160 / 10(3.6)$ & $7 / 147 / 22(4.5)$ & 0.673 \\
\hline Relevant alcohol intake ${ }^{+}$(yes/no/NA, \% yes) & $20 / 154 / 2(11.5)$ & $10 / 155 / 11(6.1)$ & 0.078 \\
\hline
\end{tabular}

Quantitative measures are expressed as mean with standard deviation or as absolute count $(\mathrm{n})$ with relative frequency (\%). Abbreviations: BMI, body mass index; NA, not available. ${ }^{+}$Alcohol intake $>12 \mathrm{~g} / \mathrm{d}$ women, $>24 \mathrm{~g} / \mathrm{d}$ men. 
Supplementary Table 1: Histologic fibrosis stages in the non-alcohol-related fatty liver disease (NAFLD) population.

\begin{tabular}{llll}
\hline & Total & PiMZ & PiMS \\
& $n=643$ & $n=36(5.6 \%)$ & $n=29(4.5 \%)$ \\
\hline Fibrosis stage F0 & 362 & $9(2.5)$ & $14(3.9)$ \\
Fibrosis stage F1 & 144 & $12(8.3)$ & $6(4.2)$ \\
Fibrosis stage F2 & 35 & $5(14.3)$ & $2(5.7)$ \\
Fibrosis stage F3 & 34 & $1(2.9)$ & $4(11.8)$ \\
Fibrosis stage F4 & 68 & $9(13.2)$ & $3(4.4)$ \\
\hline
\end{tabular}

The absolute count (n) and relative frequency (\%) of the analysed individuals are displayed. Only individuals carrying the PiMZ or PiMS genotype are displayed as there were no individuals with the PiZZ, PiSS, or PiSZ genotype. Abbreviations: PiMZ=heterozygous for the PiZ variant; PiMS=heterozygous for the PiS variant; $\mathrm{PiZZ}=$ homozygous for the $\mathrm{PiZ}$ variant; $\mathrm{PiSS}=$ homozygous for the $\mathrm{PiS}$ variant;

$\mathrm{PiSZ}=$ compound heterozygous for the $\mathrm{PiS}$ and the $\mathrm{PiZ}$ variant. 
Supplementary Table 2: Characteristics of the non-alcohol-related fatty liver disease (NAFLD) cohort.

\begin{tabular}{lllll}
\hline & Total & $\begin{array}{l}\text { Controls } \\
(\mathrm{F0}) \\
n=362(56.3 \%)\end{array}$ & $\begin{array}{l}\text { Cases } \\
(\mathrm{F4}) \\
n=68(10.6 \%)\end{array}$ & $\begin{array}{l}\text { Significance } \\
(\boldsymbol{p} \text { value })\end{array}$ \\
\hline Characteristics & $n=643$ & & & \\
Age (years) & $49 \pm 14$ & $46 \pm 13$ & $61 \pm 13$ & $<0.0001$ \\
Women & $322(50)$ & $165(46)$ & $41(60)$ & 0.026 \\
BMI (kg/m²) & $37 \pm 12$ & $36 \pm 12$ & $28 \pm 7$ & $<0.0001$ \\
Diabetes mellitus & $188 / 403 / 52(32)$ & $84 / 265 / 13(24)$ & $24 / 14 / 30(63)$ & $<0.0001$ \\
(yes/no/NA, \% yes) & & & & \\
& & & & \\
Frequency of AAT & & & & \\
genotypes & $34(5.3)$ & $9(2.5)$ & $9(13.2)$ & $<0.0001$ \\
PiMZ genotype & $0(0)$ & $0(0)$ & $0(0)$ & $\mathrm{NA}$ \\
PiZZ genotype & $29(4.5)$ & $14(3.9)$ & $3(4.4)$ & $0.740^{*}$ \\
PiMS genotype & $0(0)$ & $0(0)$ & $0(0)$ & $\mathrm{NA}$ \\
PiSS genotype & $0(0)$ & $0(0)$ & $0(0)$ & $\mathrm{NA}$ \\
PiSZ genotype & & & \\
\hline
\end{tabular}

Cases were defined as NAFLD patients with histological cirrhosis (stage F4) whereas controls were defined as NAFLD patients without histological evidence of fibrosis (stage F0). Quantitative measures are shown as mean \pm standard deviation or as an absolute count $(n)$ and relative frequency $(\%)$.

Abbreviations: BMI, body mass index; NA, not available/applicable; PiMZ=heterozygous for the PiZ variant; PiZZ=homozygous for the PiZ variant; PiMS=heterozygous for the PiS variant; PiSS=homozygous for the PiS variant; PiSZ=compound heterozygous for the PiS and the PiZ variant. "Fisher exact test. 
Supplementary Table 3: Characteristics of German-Swiss cohort of alcohol misusers.

\begin{tabular}{|c|c|c|c|c|}
\hline & $\begin{array}{l}\text { Total } \\
n=1798\end{array}$ & $\begin{array}{l}\text { Controls } \\
\text { (no liver injury) } \\
n=864(48.1 \%) \\
\end{array}$ & $\begin{array}{l}\text { Cases } \\
\text { (cirrhosis) } \\
n=934(51.9 \%)\end{array}$ & $\begin{array}{l}\text { Significance } \\
\text { ( } p \text { value) }\end{array}$ \\
\hline \multicolumn{5}{|l|}{ Characteristics } \\
\hline Age (years) & $51 \pm 11$ & $45 \pm 10$ & $56 \pm 10$ & $<0.0001$ \\
\hline Women & $386(22)$ & $118(14)$ & $268(29)$ & $<0.0001$ \\
\hline $\mathrm{BMI}\left(\mathrm{kg} / \mathrm{m}^{2}\right)$ & $25.6 \pm 4.6$ & $24.9 \pm 4.9$ & $26.3 \pm 5.0$ & $<0.0001$ \\
\hline $\begin{array}{l}\text { Diabetes mellitus } \\
\text { (yes/no/NA, \% yes) }\end{array}$ & 209/1199/390 (15) & 13/612/239 (2) & 196/587/151 (25) & $<0.0001$ \\
\hline \multicolumn{5}{|l|}{$\begin{array}{l}\text { Frequency of AAT } \\
\text { genotypes }\end{array}$} \\
\hline PiMZ genotype & $75(4.17)$ & 17 (1.97) & $58(6.21)$ & $<0.0001$ \\
\hline PiZZ genotype & $4(0.12)$ & $0(0)$ & $4(0.43)$ & $0.073^{*}$ \\
\hline PiMS genotype $^{+}$ & $120(6.77)$ & 47 (5.53) & 73 (7.92) & 0.046 \\
\hline PiSS genotype $^{+}$ & $3(0.17)$ & $1(0.06)$ & $2(0.11)$ & $0.390^{*}$ \\
\hline PiSZ genotype $^{+}$ & $6(0.34)$ & $0(0)$ & $6(0.65)$ & $0.019^{*}$ \\
\hline
\end{tabular}

Cases were defined as alcohol misusers with cirrhosis whereas controls were defined as alcohol misusers without evidence of significant liver injury. Quantitative measures are shown as mean \pm standard deviation or as an absolute count (n) and relative frequency (\%). Abbreviations: BMI, body mass index; NA, not available; PiMZ=heterozygous for the PiZ variant; PiZZ=homozygous for the PiZ variant; PiMS=heterozygous for the PiS variant; PiSS=homozygous for the PiS variant; $\mathrm{PiSZ}=$ compound heterozygous for the PiS and the PiZ variant. +1772 subjects ( $98.6 \%$ of all subjects) were genotyped for presence of the PiS variant. *Fisher exact test. 
Supplementary Table 4: Characteristics of British-Irish cohort of alcohol misusers.

\begin{tabular}{|c|c|c|c|c|}
\hline & $\begin{array}{l}\text { Total } \\
n=664\end{array}$ & $\begin{array}{l}\text { Controls } \\
\text { (no liver injury) } \\
n=347(52.2 \%)\end{array}$ & $\begin{array}{l}\begin{array}{l}\text { Cases } \\
\text { (cirrhosis) } \\
n=317(47.8 \%)\end{array} \\
\end{array}$ & $\begin{array}{l}\text { Significance } \\
\text { ( } p \text { value) }\end{array}$ \\
\hline \multicolumn{5}{|l|}{ Characteristics } \\
\hline Age (years) & $51 \pm 11$ & $49 \pm 10$ & $53 \pm 10$ & $<0.0001$ \\
\hline Women & $180(27)$ & $81(23)$ & $99(31)$ & 0.022 \\
\hline BMI $\left(\mathrm{kg} / \mathrm{m}^{2}\right)^{\circ}$ & $24.7 \pm 2.5$ & $24.7 \pm 2.3$ & $24.6 \pm 2.6$ & 0.901 \\
\hline $\begin{array}{l}\text { Diabetes mellitus } \\
\text { (yes/no/NA, \% yes) }^{\circ}\end{array}$ & - & - & - & - \\
\hline \multicolumn{5}{|l|}{$\begin{array}{l}\text { Frequency of AAT } \\
\text { genotypes }\end{array}$} \\
\hline PiMZ genotype & $28(4.22)$ & 9 (2.59) & $19(5.99)$ & 0.029 \\
\hline PiZZ genotype & $0(0)$ & $0(0)$ & $0(0)$ & NA \\
\hline PiMS genotype & $61(9.30)$ & $28(8.16)$ & $33(10.54)$ & 0.295 \\
\hline PiSS genotype $^{+}$ & $2(0.30)$ & $1(0.29)$ & $1(0.32)$ & $0.499^{*}$ \\
\hline PiSZ genotype + & $2(0.30)$ & $1(0.29)$ & $1(0.32)$ & $0.499^{*}$ \\
\hline
\end{tabular}

Cases were defined as alcohol misusers with cirrhosis whereas controls were defined as alcohol misusers without evidence of significant liver injury. Quantitative measures are shown as mean \pm standard deviation or as an absolute count ( $\mathrm{n}$ ) and relative frequency (\%). Abbreviations: BMI, body mass index;

$\mathrm{PiMZ}=$ heterozygous for the PiZ variant; PiZZ=homozygous for the PiZ variant; PiMS=heterozygous for the PiS variant; $\mathrm{PiSS}=$ homozygous for the PiS variant; $\mathrm{PiSZ}=$ compound heterozygous for the $\mathrm{PiS}$ and the $\mathrm{PiZ}$ variant; NA, not applicable. ${ }^{\circ}$ Diabetic subjects and/or subjects with $\mathrm{BMI}>30 \mathrm{~kg} / \mathrm{m}^{2}$ have been excluded $a$ priori. "Patients with the PiZZ genotype were excluded a priori. ${ }^{+} 656$ subjects $(98.8 \%$ of all subjects) were genotyped for presence of the PiS variant. *Fisher exact test. 


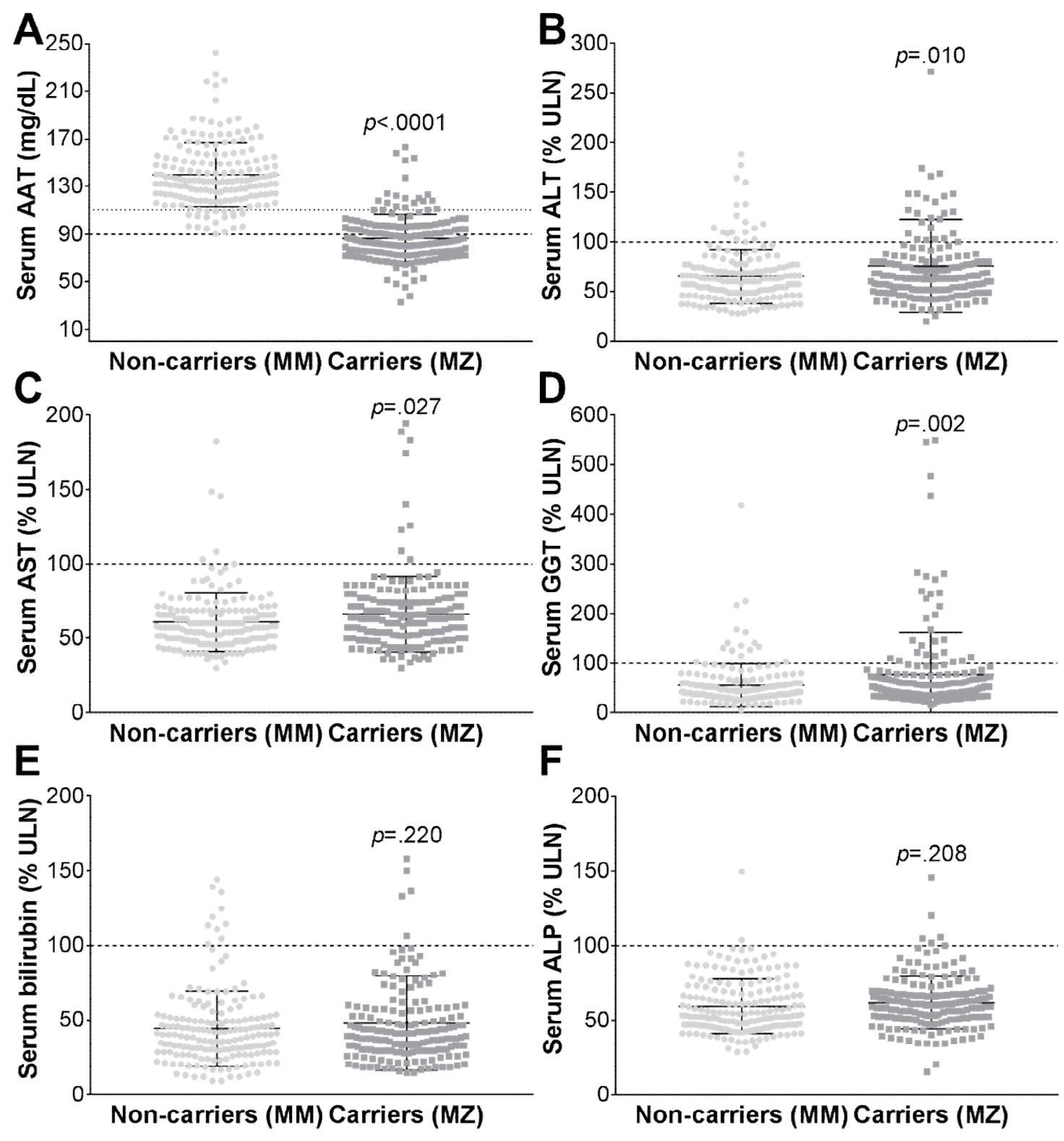

Figure 1: Serum alpha1-antitrypsin (AAT) and liver enzyme concentrations in carriers heterozygous for the alpha1-antitrypsin PiZ variant (MZ) and non-carriers (MM).

176 carriers and 176 non-carriers undertook laboratory analysis. A) Scatter plot of the absolute serum AAT concentrations: The lower dotted line represents the commonly used lower limit of normal (90 $\mathrm{mg} / \mathrm{dL}$ ). The upper dotted line represents the $110 \mathrm{mg} / \mathrm{dL}$ cut-off level which was previously suggested to better discriminate between individuals with the PiMZ and PiMM genotype. B-F) Scatter plots depict liver enzyme activities as well as cholestatic markers normalized to the sex-specific upper limit of normal (ULN) which is marked as a dotted line. Abbreviations: MM, wild-type genotype (PiMM); MZ, heterozygous carriage of the PiZ variant (PiMZ); ALT, alanine aminotransferase, AST, aspartate aminotransferase; GGT, gamma-glutamyl transferase; ALP, alkaline phosphatase. 

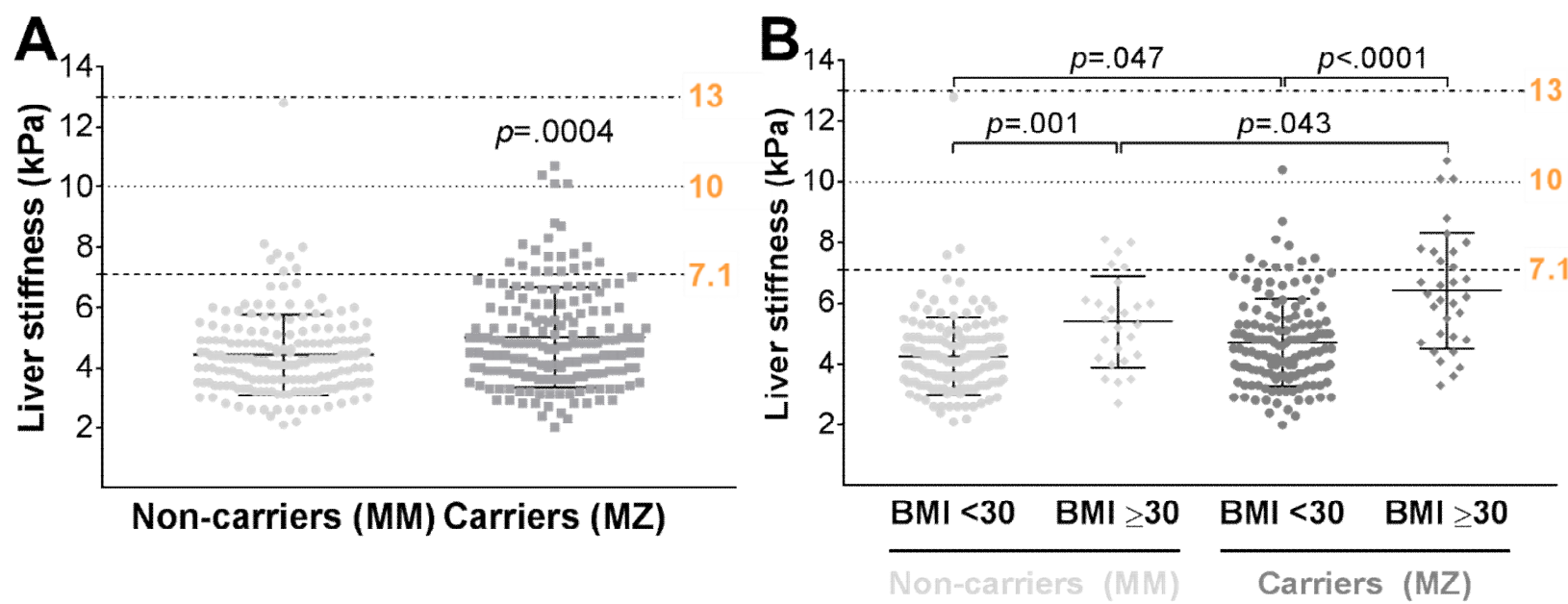

Figure 2: Liver stiffness evaluated by transient elastography in carriers heterozygous for the alpha1-antitrypsin PiZ variant (MZ) and non-carriers (MM).

176 carriers and 176 non-carriers without a previously known liver disease have been prospectively evaluated by transient elastography (FibroScan, Echosens). The horizontal dotted lines indicate the established liver stiffness cut-off levels associated with increasing degrees of hepatic fibrosis, namely: fibrosis stage $\geq 2-7.1 \mathrm{kPa}$, fibrosis stage $\geq 3-10 \mathrm{kPa}$, fibrosis stage $4-13 \mathrm{kPa}$. Abbreviations: $\mathrm{MM}$, wildtype genotype (PiMM); MZ, heterozygous carriage of the PiZ variant (PiMZ); BMI, body mass index. 


\section{A) Unadjusted odds ratios}

Cirrhosis (y/n) $\longmapsto-5.76[1.82-18.24](p=.003)$

$\begin{array}{ccccccccc} & T & 1 & 1 & 1 & 1 & 1 & 1 \\ \text { OR } & 1 & 5 & 10 & 15 & 20 & 25 & 30\end{array}$

\section{B) Adjusted odds ratios}

\section{Cirrhosis $(y / n)$}

6.73 [1.46-30.99] $(p=.014)$

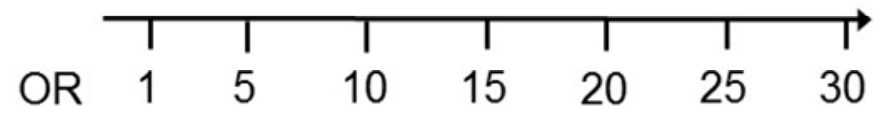

Figure 3: Risk of developing cirrhosis in biopsy-proven NAFLD patients heterozygous for the alpha1-antitrypsin PiZ variant.

An univariate $(A)$ and a multivariate $(B)$ analysis in 378 patients with non-alcoholic fatty liver disease (NAFLD) from Germany and Austria, the latter was adjusted for sex, age, body mass index, and the presence of diabetes mellitus. The odds for developing cirrhosis (stage F4) vs. no fibrosis (stage F0) are displayed. Abbreviations: OR, odds ratio; $y$, yes; $n$, no. 


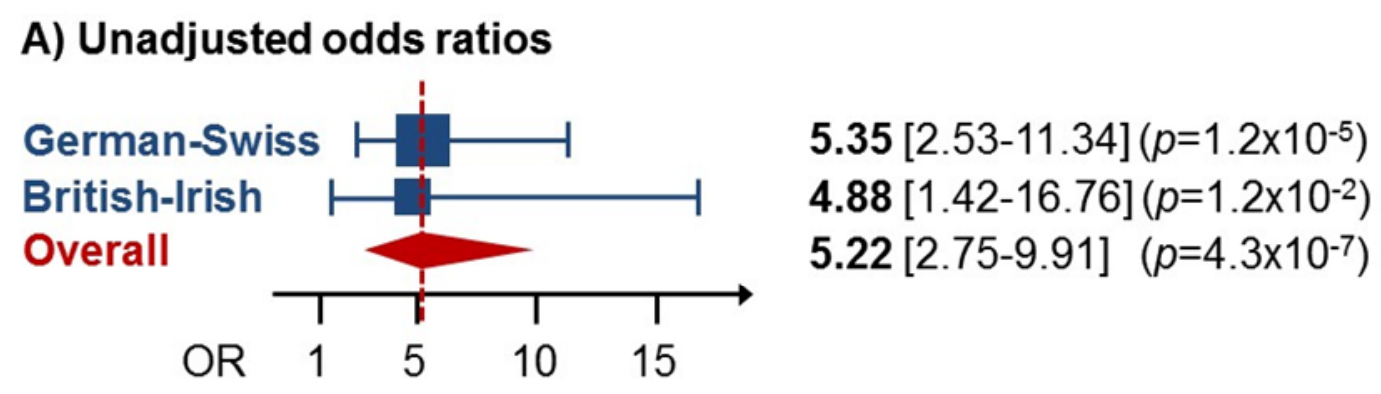

\section{B) Adjusted odds ratios}

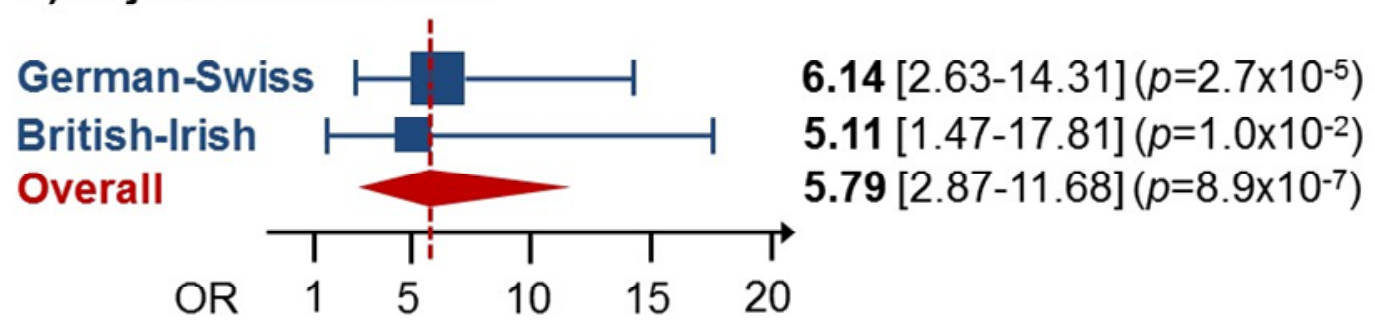

Figure 4: Analysis of the risk of developing cirrhosis in two cohorts of alcohol misusers heterozygous for the alpha1-antitrypsin PiZ variant.An univariate $(A)$ and a multivariate $(B)$ analysis was performed in 1191 alcohol misusers from Germany and Switzerland as well as 440 alcohol misusers from Great Britain and Ireland, both cohorts with and without cirrhosis. Adjustments were made for sex, age, body mass index, and the presence of diabetes mellitus. The term "overall" depicts the meta-analysis of both cohorts. Abbreviations: OR, odds ratio. 
A) Unadjusted odds ratios

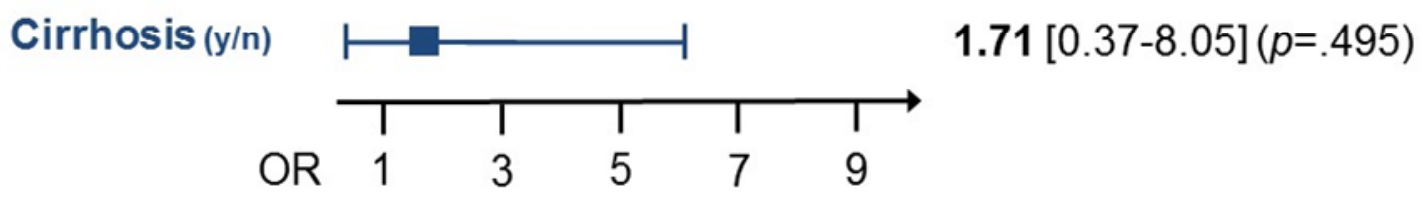

\section{B) Adjusted odds ratios}

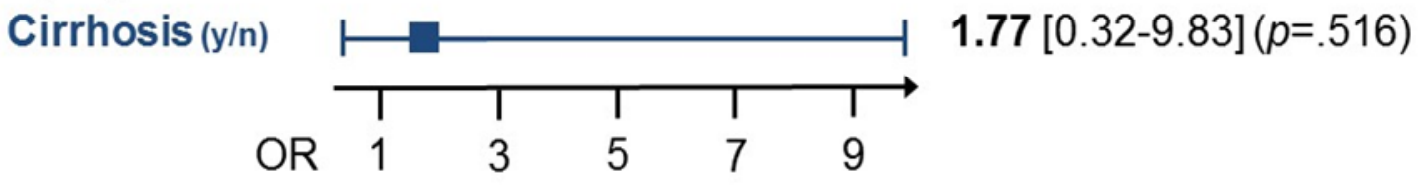

Supplementary figure 1: Odds for cirrhosis development in biopsy-proven NAFLD individuals carrying the alpha1-antitrypsin PiS variant.

An univariate $(A)$ and a multivariate $(B)$ analysis was performed in 378 patients with non-alcoholic fatty liver disease (NAFLD) from Germany and Austria, the latter was adjusted for sex, age, body mass index, and the presence of diabetes mellitus. The odds for developing liver cirrhosis (stage F4) vs. no fibrosis (stage F0) are displayed. Abbreviations: OR, odds ratio; y, yes; n; no. 


\section{A) Unadjusted odds ratios}

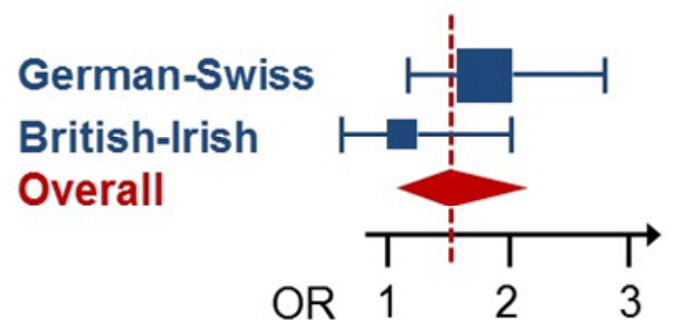

$1.79[1.15-2.79](p=.010)$

$1.10[0.60-2.01](p=.750)$

$1.51[1.06-2.15](p=.024)$

\section{B) Adjusted odds ratios}

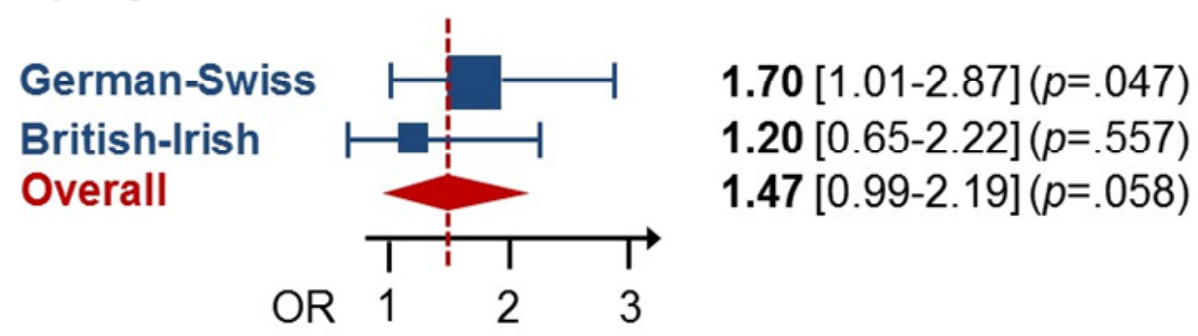

Supplementary figure 2: Analysis of the risk of developing cirrhosis in two cohorts of alcohol misusers carrying the alpha1-antitrypsin PiS variant.

An univariate $(A)$ and a multivariate $(B)$ analysis was performed in 1176 alcohol misusers from Germany and Switzerland as well as 437 alcohol misusers from Great Britain and Ireland, both cohorts with and without cirrhosis. Adjustments were made for sex, age, body mass index, and the presence of diabetes mellitus. The term "overall" depicts the meta-analysis of both cohorts. Abbreviations: OR, odds ratio. 